\title{
Compressed Sensing for OFDM UWB Systems
}

\author{
Tanish Agrawal ${ }^{* \dagger}$, Vishwas Lakkundi*, Anthony Griffin*, and Panagiotis Tsakalides* \\ *Institute of Computer Science, Foundation for Research and Technology-Hellas, Greece \\ $\dagger$ Advanced Learning and Research Institute, University of Lugano, Switzerland
}

\begin{abstract}
This paper considers compressed sensing (CS) techniques for signal reconstruction and channel estimation in OFDM-based high-rate ultra wideband (UWB) communication systems. We employ a parallel CS structure that exploits frequency domain sparsity. We also consider multipath UWB channels in both the line-of-sight and non line-of-sight environments. UWB signal detection and channel estimation from sub-Nyquist analog projections is carried out using an optimized orthogonal matching pursuit algorithm and the smoothed $\ell_{0}$ algorithm. Simulation results demonstrate significant gains in the form of reliable signal recovery and channel estimation as well as dramatically sub-Nyquist sampling rates for the analog-to-digital converters while maintaining high data rates.
\end{abstract}

Index Terms-Channel estimation, compressed sensing, multipath channels, OFDM, signal reconstruction, UWB.

\section{INTRODUCTION}

As with any wideband communications, sampling is a major bottleneck for ultra wideband (UWB) systems. Due to the wide spectral range of multiband signals, their Nyquist rates easily exceed the specifications of available analog-todigital converters (ADCs) by orders of magnitude. Moreover, conventional spectral estimation methods that operate at or above the Nyquist rate pose a major implementation challenge. Meanwhile, traditional linear signal reconstruction methods, due to their timing requirements for rapid sensing, enable acquisition of only a limited number of measurements from the received signal, providing insufficient statistics.

By employing compressed sensing (CS) techniques [1], [2], sparse signals can be recovered, with a high probability, from a set of random linear projections - known as measurementsusing non-linear reconstruction algorithms. Typically, the number of random measurements is much lower than the number of samples in the original signal, thus leading to a significant reduction in sampling rates. Reduced sampling rates directly translate into reduced number of required ADC resources, which is of great importance for UWB systems.

Previously, various CS techniques have been explored to address signal reconstruction, channel estimation, and symbol detection issues for only the impulse radio version of UWB [3], [4]. A parallel wideband structure applying CS to analog signals directly has been proposed in [5] to successfully reconstruct orthogonal frequency division multiplexing (OFDM) signals in cognitive radio assuming perfect channel

This work was funded by the Marie Curie TOK-DEV "ASPIRE" (MTKDCT-2005-029791) and IAPP "CS-ORION" (PIAP-GA-2009-251605) grants within the 6th and 7th Framework Programs of the European Community. state information (CSI). But, the issues concerning complexvalued non-sparse OFDM-based UWB signals and the associated multipath channels have not been considered so far to the best of our knowledge. In this paper, we specifically address these OFDM UWB signal reconstruction and channel estimation challenges using CS relying on recently developed ideas. Additionally, we consider the presence of real-world multipath UWB channels as well. Our simulations demonstrate that OFDM UWB signals can be successfully reconstructed and channels adequately estimated even in the presence of multipath channels at sub-Nyquist sampling rates using CS. We also analyze and compare different CS algorithms in terms of performance and complexity.

The rest of this paper is organized as follows. Salient features of the high-rate UWB system and CS techniques are outlined in Section II. Section III describes the signal reconstruction and channel estimation procedures. Simulation results are presented in Section IV and finally, conclusions are drawn in Section V.

\section{Compressed Sensing of OFDM UWB Signals}

\section{A. Ultra Wideband Review}

UWB is an emerging radio technology operating across a wide range of unlicensed frequency spectrum $(3.1-10.6 \mathrm{GHz})$ that enables very high data transfer rates over short distances. The ECMA-368 standard for high data rate UWB systems published in December 2008 incorporates enhanced PHY and MAC layer specifications based on multiband OFDM. The UWB spectrum is divided into 14 bands, each with a bandwidth of $528 \mathrm{MHz}$. A total of 112 subcarriers are used per band (100 data and 12 pilot subcarriers) that allow coherent detection. A symbol is formed by adding an OFDM symbol (128-point IFFT output) and a zero-padded suffix (ZP) of 37 samples. The standard also recommends that a device has to transmit at least 86 useful tones per symbol to ensure an optimum receive performance. Each packet consists of 6 OFDM symbols for channel estimation and a variable length payload (0 - 4095 octets).

The indoor channels for UWB are based on the modified Saleh-Valenzuela model [6], which assumes that multipath components arrive in clusters described by a statistically random Poisson process. Four UWB channel models are defined each with either a line-of-sight (LOS) or non line-of-sight (NLOS) environment and varying range: CM1 (LOS, 0-4m), CM2 (NLOS, 0-4m), CM3 (NLOS, 4-10m), CM4 (extreme NLOS) [7]. 


\section{B. Compressed Sensing Background}

CS-based signal acquisition is more efficient than traditional sampling for sparse or compressible signals [8]. Let $x(t)$ be an analog signal that is sampled at the Nyquist rate and processed in frames of $N$ samples. Each frame is then an $N \times 1$ vector $\boldsymbol{x}$ given by $\boldsymbol{x}=\boldsymbol{\Psi} \boldsymbol{X}$, where $\boldsymbol{\Psi}$ is an $N \times N$ matrix whose columns are the similarly-sampled basis functions $\Psi_{i}(t)$, and $\boldsymbol{X}$ is the vector that chooses the linear combinations of the basis functions. $\boldsymbol{X}$ can be thought of as $\boldsymbol{x}$ in the domain of $\boldsymbol{\Psi}$, and it is $\boldsymbol{X}$ that is required to be sparse for CS to perform well. We say that $\boldsymbol{X}$ is $K$-sparse if it contains only $K$ nonzero elements. In other words, $\boldsymbol{x}$ can be exactly represented by the linear combination of $K$ basis functions.

CS theory states that $x$ can be reconstructed successfully from $M$ measurements, where $M \ll N$. The measurement process is carried out by projecting $\boldsymbol{x}$ over another random basis $\Phi$ that is incoherent with $\Psi$. Thus the measurements are represented by the $M \times 1$ vector $\boldsymbol{y}$ given by $\boldsymbol{y}=\boldsymbol{\Phi} \boldsymbol{x}=\boldsymbol{\Phi} \boldsymbol{\Psi} \boldsymbol{X}$. For signal reconstruction, CS employs convex optimization techniques such as $\ell_{1}$-norm minimization as defined below:

$$
\hat{\boldsymbol{X}}=\arg \min \|\boldsymbol{X}\|_{1} \quad \text { s.t. } \quad \boldsymbol{y}=\boldsymbol{\Phi} \boldsymbol{\Psi} \boldsymbol{X} .
$$

The optimization problem in (1) can be solved by techniques involving linear programming such as basis pursuit or nonlinear iterative greedy algorithms such as orthogonal matching pursuit (OMP) [9].

\section{Parallel Segmented Compressed Sensing}

Signal processing in wideband systems requires high-speed ADCs operating at the Nyquist sampling rate. This requirement can be avoided by applying CS to the analog signal directly. Analog-to-information conversion (AIC) described in [10] is one such approach.

However, the AIC approach requires the use of a high order analog filter to achieve optimum signal reconstruction. In addition, the serially-obtained measurements-which are no longer independent due to the convolution in the filterintroduce some redundancy. A parallel version of AIC called parallel segmented compressed sensing (PSCS) is proposed in [5] and its structure is illustrated in Fig. 1. The received signal is sliced up into $S$ segments $T_{s}$ seconds long, and the random projection is applied to each segment separately through $P$ parallel branches, thus generating $M=S P$ measurements every $T$ seconds. The measurement of the $s$-th segment of the $p$-th branch is then given by

$$
\begin{aligned}
y_{s P+p} & =\left\langle r_{s}(t), \Phi_{s P+p}(t)\right\rangle \\
& =\int_{s T_{s}}^{(s+1) T_{s}} r(t) \Phi_{s P+p}^{*}(t) d t
\end{aligned}
$$

where $\Phi_{s P+p}(t)$ is chosen randomly for all $s$ and $p$ from a Bernoulli distribution and the inner product is implemented with mixers and integrators. The outputs of the integrators are fed to a set of parallel ADCs at the end of each integration time $T$ and the quantized digital data are sent for further digital signal processing. Each parallel branch samples at a

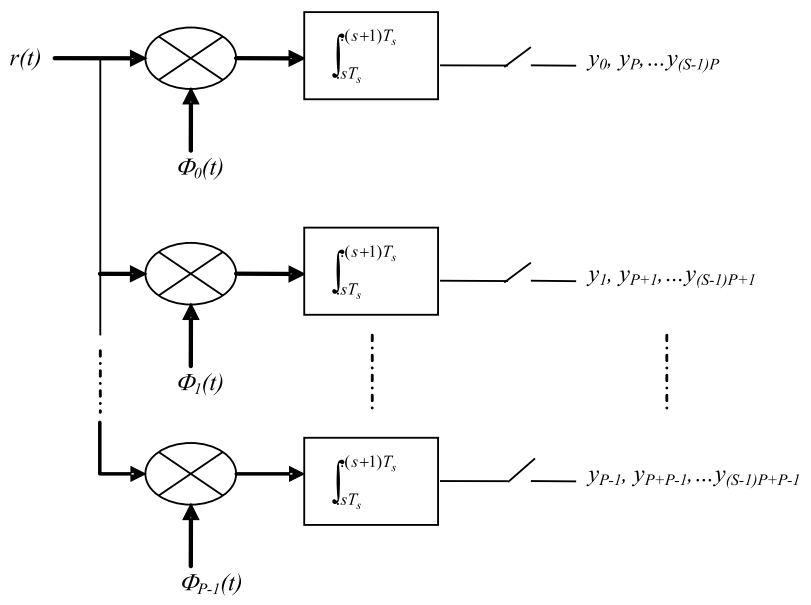

Fig. 1. Parallel segmented compressed sensing from [5]

sub-Nyquist rate as long as $T>T_{N}$, where $T_{N}$ is the Nyquist sampling period.

The signal $\boldsymbol{x}$ is then reconstructed using a modified version of OMP by processing all $M$ measurements jointly. The interested reader is referred to [5] for more details. Thus the ADC on each branch takes $S$ samples per frame, where $S \ll N$, which results in much lower demands on the ADCs for a moderate increase in complexity. This makes PSCS particularly interesting for wideband systems such as OFDM UWB.

However, the work of [5] only considered extremely sparse signals with perfect CSI. In the following section, we propose an extended PSCS scheme that considers signals that are not sparse and also address the problem of channel estimation for OFDM UWB.

\section{Signal Recovery and Channel Estimation}

\section{A. Non-sparse Signal Reconstruction}

We were interested in exploring whether PSCS could be used in a real OFDM UWB standard, namely, the ECMA 368 standard [11] as discussed in Section II-A. Additionally, we wanted to apply real channel models to PSCS and to explore the use of less sparse signals, as decreasing the sparsity of an OFDM signal equates to increasing the throughput, which is of course highly desirable in high-rate UWB systems.

Fig. 2 shows the bit error rate (BER) performance vs the bit energy to noise power ratio $\left(E_{b} / N_{0}\right)$ in additive white Gaussian noise (AWGN). The OFDM symbols follow the ECMA 368 standard, but out of the 112 usable subcarriers, only $K$ are in use (12 pilots and $K-12$ data). The curve labeled "Nyquist" samples the signal at the Nyquist rate (165 samples per symbol), and represents the performance standard that we want to achieve. The PSCS with $K=32$ curve uses 10 segments and 16 branches for a total of 160 measurements per symbol, and it is clear that it is unusable at this level of sparsity, due to the irreducible error rate at about $2.5 \times 10^{-3}$.

In order to improve the BER performance, we propose extended PSCS (EPSCS), in which the OMP algorithm operates 


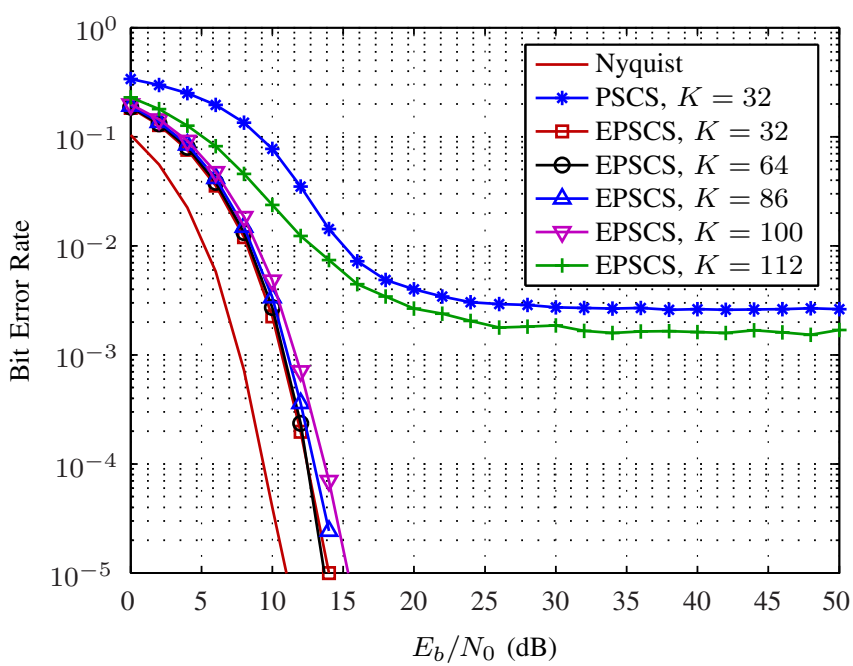

Fig. 2. BER vs $E_{b} / N_{0}$ for the AWGN channel, with $K$ subcarriers. The curve labeled "Nyquist" is the performance of Nyquist sampling. The other curves use 10 segments and 16 branches and PSCS and EPSCS as indicated.

knowing the location of the subcarriers used in each symbol as shown in Algorithm 1. This modification dramatically increases the BER performance as is illustrated by the EPSCS curve in Fig. 2. In fact, EPSCS allows up to 100 subcarriers to be used without significant degradation when compared to the Nyquist-sampled case. This is a very important result as it allows much more data to be successfully recovered when using EPSCS.

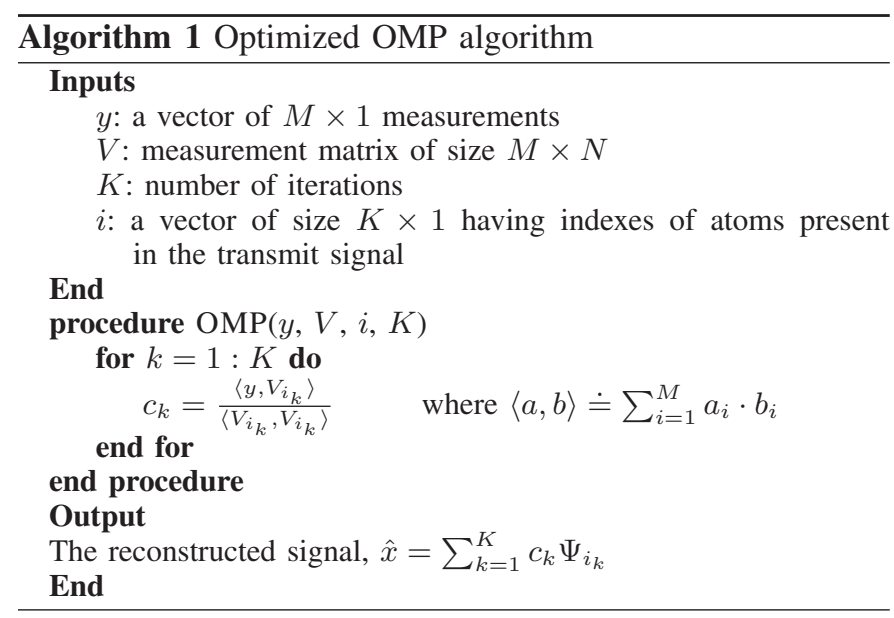

The extra constraint of the receiver knowing the subcarriers that are used is not particularly burdensome, and there are two ways to deal with this. The first is to simply use a fixed subset of carriers which would require a trivial amount of overhead. The second-which would marginally improve results in multipath channels-is to randomly choose the subset of subcarriers in the transmitter and pass the seed of the random sequence to the receiver in the preamble sequence. EPSCS also allows a significant reduction in complexity, as the OMP algorithm is more efficient when the location of the subcarriers is known.

\section{B. UWB Channel Estimation using CS}

The channel realizations provided by the IEEE for UWB as defined in [7] are used here to simulate the effects of multipath channels. The time domain signal $x(t)$ is passed through one of the channel models and the measurements are obtained through the EPSCS structure described earlier. Hence, assuming perfect synchronization, the received signal $r_{h}(t)$ in the presence of the multipath channel $h(t)$ can be written as

$$
r_{h}(t)=x(t) * h(t)+n(t),
$$

where $*$ denotes convolution. We assume that the channel is constant for each packet of data, and during the channel estimation sequence, we transmit known values on each of the $K$ subcarriers chosen for that packet. As the main motive here is to compare the performance of the signal recovered through EPSCS against the Nyquist case, a simple least squares estimator given in [12] is used for channel estimation. The six channel estimation symbols are recovered by the modified OMP algorithm in the same way as the data symbols. The channel frequency response at the $K$ positions is calculated by dividing the estimated signal by the known sequence, averaged over six channel estimation symbols. Channel estimation can be further improved by using the pilots among the data symbols. The performance of the channel estimation is presented and discussed in the following section, with simulation results.

\section{Simulation Results}

We only considered channel models CM1 and CM2, as CM3 and CM4 would require more sophisticated channel estimation and equalisation, which was beyond the scope of this work. Due to lack of space we present only results involving CM2 here, however the CM1 results are very similar.

The BER performance of the channel estimation for CM2 is shown in Fig. 3. The EPSCS curves use 10 segments and 16 parallel branches, and the indicated number of subcarriers. Similar to the AWGN case shown in Fig. 2, the "Nyquist" curve represents the best possible performance that EPSCS could hope to achieve. It is again clear that EPSCS performs very well, except when 112 subcarriers are used. This is a very encouraging result as it demonstrates that EPSCS can support non-sparse signals in the presence of non-perfect CSI.

We then took the case of EPSCS with 86 subcarriersthe minimum recommended by the ECMA-368 standard-and investigated the effects of varying $S$ and $P$. Fig. 4 presents the performance for CM2. It is clear that 14 is the minimum number of branches required when 10 segments are used. The performance of EPSCS remains constant when $S$ and $P$ are changed from 10 and 16 to 20 and 8 , respectively, indicating a flexibility in the selection of $S$ vs $P$.

As $S$ determines the sampling rate for each ADC, and the Nyquist sampling rate is 165 samples per symbol, the values 10 and 20 obtained due to EPSCS correspond to just $6 \%$ and $12 \%$ of the Nyquist rate.

Furthermore, we compared the performance of OMP against the smoothed $\ell_{0}$ (SL0) reconstruction algorithm, which tries 


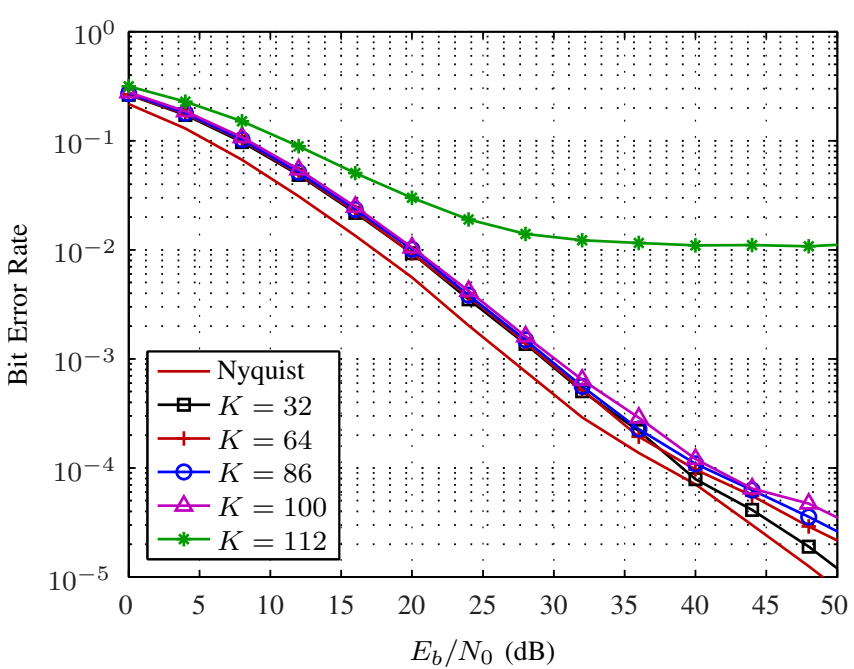

Fig. 3. BER vs $E_{b} / N_{0}$ using OMP and CM2 with channel estimation, for varying numbers of used sub-carriers $K$. The curve labeled "Nyquist" is the performance of Nyquist sampling, which is independent of $K$. The other curves use EPSCS with $S=10, P=16$, and the indicated value of $K$.

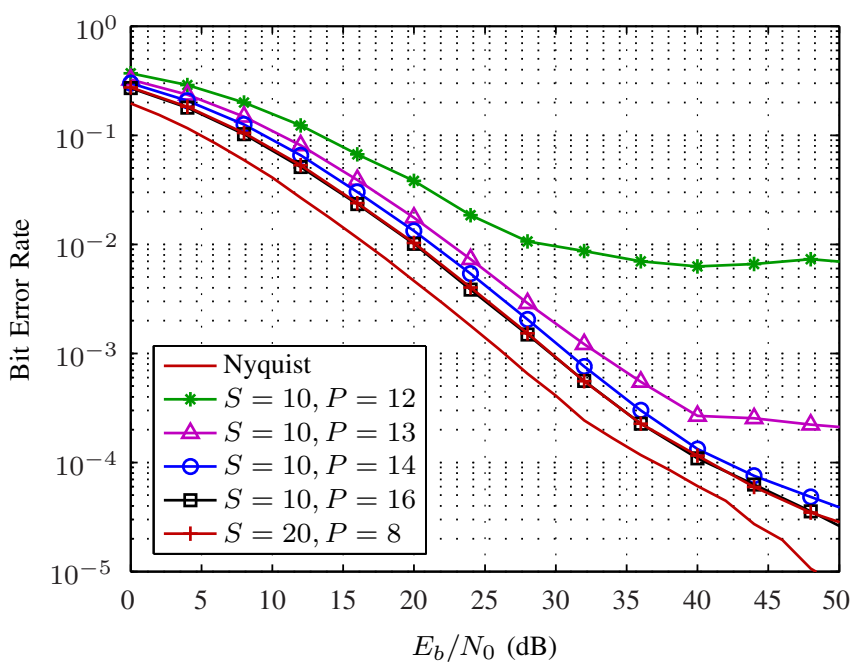

Fig. 4. BER vs $E_{b} / N_{0}$ using OMP and CM2 with channel estimation, for varying values of $S$ and $P$. The curve labeled "Nyquist" is the performance of Nyquist sampling, which is independent of $S$ and $P$. The other curves use EPSCS with $K=86$, and the indicated values of $S$ and $P$.

to approximate the $\ell_{0}$-norm by a smooth function and uses gradient-based methods for its minimization [13].

Fig. 5 shows BER performance when the signal is reconstructed using the SLO algorithm without knowing the subcarrier indices. The curves for $K=32$ and 64 follow the Nyquist curve very closely indicating a very good performance for sparse signals. The performance deteriorates for less sparse signals ( $K=86$ and 100$)$ as indicated by error floors. In terms of time complexity, SLO has the advantage of being faster than OMP especially when sparsity $K>40$.

\section{Conclusions}

In this paper, we have proposed EPSCS that relies on recent advances in compressed sensing for OFDM by adapting

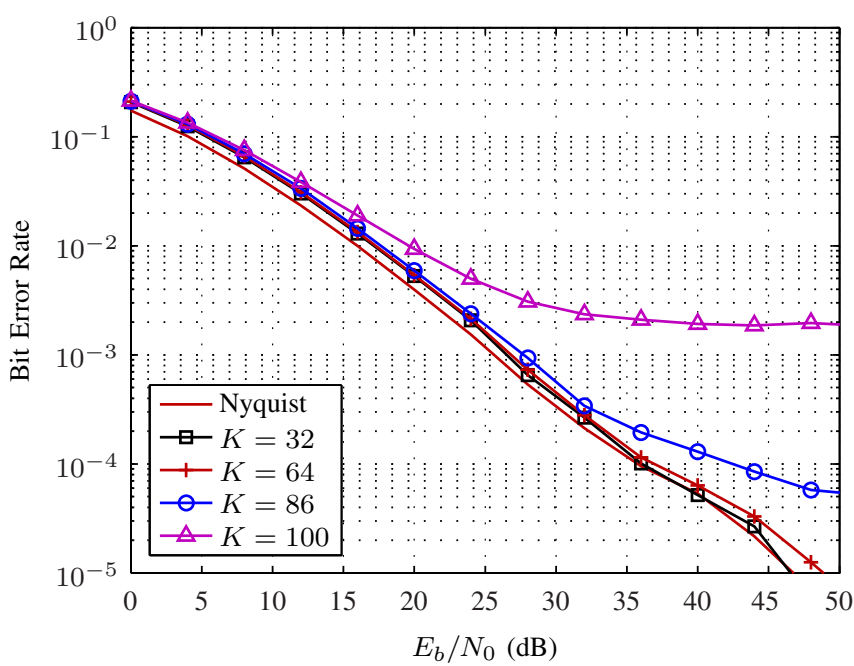

Fig. 5. BER vs $E_{b} / N_{0}$ using SLO and CM2 with channel estimation, for varying numbers of used sub-carriers $K$. The curve labeled "Nyquist" is the performance of Nyquist sampling, which is independent of $K$. The other curves use EPSCS with $S=10, P=16$, and the indicated value of $K$.

it to non-sparse signals and applying it to realistic channel models for high-rate OFDM-based UWB systems. The results indicate that it is possible to successfully recover high-rate data using significantly reduced sampling rate constraints on ADCs, which is of particular interest in UWB communications. The selection of a suitable reconstruction algorithm depends on the trade-off between performance and complexity.

\section{REFERENCES}

[1] E. Candes and M. Wakin, "An introduction to compressive sensing," IEEE Sig. Proc. Mag., pp. 21-30, March 2008.

[2] D. Donoho, "Compressed sensing," IEEE Trans. Inform. Theory, vol. 52, no. 4, pp. 1289-1306, April 2006.

[3] J. L. Paredes, G. R. Arce, and Z. Wang, "Ultra-wideband compressed sensing: channel estimation," IEEE J. Sel. Topics in Sig. Proc., vol. 1, no. 3, pp. 383-395, October 2007.

[4] P. Zhang, Z. Hu, R. Qiu, and B. Sadler, "A compressed sensing based ultra-wideband communication system," in Proc. IEEE Int. Conf. on Comm. (ICC), Dresden, Germany, June 2009.

[5] Z. Yu, S. Hoyos, and B. M. Sadler, "Mixed signal parallel compressed sensing and reception for cognitive radio," in Proc. IEEE Int. Conf. on Acoustics, Speech, and Signal Processing (ICASSP), Las Vegas, Nevada, USA, April 2008.

[6] A. Saleh and R. Valenzuela, "A statistical model for indoor multipath propagation," IEEE J. Select. Areas Commun., vol. 5, no. 2, pp. 128-137, February 1987.

[7] J. R. Foerster, "Channel modeling sub-committee report (final)," IEEE P802.15-02/490r1-SG3a, 2003.

[8] R. G. Baraniuk, "Compressive sensing," IEEE Sig. Proc. Mag., pp. 118120, July 2007.

[9] J. A. Tropp and A. C. Gilbert, "Signal recovery from random measurements via orthogonal matching pursuit," IEEE Trans. Inform. Theory, vol. 53, no. 12, pp. 4655-4666, December 2007.

[10] J. N. Laska, S. Kirolos, R. G. Baraniuk, and et al, "Theory and implementation of an analog-to-information converter using random demodulation," in Proc. IEEE Int. Symp. on Circ. and Sys. (ISCAS), New Orleans, Louisiana, USA, May 2007.

[11] ECMA-368, "High rate UWB PHY and MAC standard," 2008.

[12] Y. Shen and E. Martinez, "Channel estimation in OFDM systems," Freescale Semiconductor Application Note, 2006.

[13] G. Mohimani, M. Babaie-Zadeh, and C. Jutten, "Complex-valued sparse representation based on smoothed $\ell^{0}$ - norm," in Proc. IEEE Int. Conf. on Acoustics, Speech, and Signal Processing (ICASSP), Las Vegas, Nevada, USA, March 2008, pp. 3881-3884. 